\title{
Cyr61 Expression Pattern and Association with Clinico- pathological Factors in Patients with Cervical Cancer
}

\author{
SEBASTIAN MAYER ${ }^{1,2}$, BORIS GABRIEL $^{3}$, THALIA ERBES ${ }^{1,2}$, SYLVIA TIMME-BRONSERT $^{2,4}$, \\ MARKUS JÄGER ${ }^{1,2}$, GERTA RÜCKER ${ }^{2,5}$, FRANCISKA KUF ${ }^{1,2}$, JIRI BOUDA ${ }^{6}$, ALENA BARTAKOVA ${ }^{6}$, \\ AXEL ZUR HAUSEN ${ }^{7}$, ELMAR STICKELER ${ }^{8}$, GERALD GITSCH $^{1,2}$ and MARC HIRSCHFELD ${ }^{1,2,9}$ \\ ${ }^{1}$ Department of Obstetrics and Gynecology, Medical Center - University of Freiburg, Freiburg, Germany; \\ ${ }^{2}$ Faculty of Medicine, University of Freiburg, Freiburg, Germany; \\ ${ }^{3}$ Department of Obstetrics and Gynecology, St. Josefs-Hospital, Wiesbaden, Germany; \\ ${ }^{4}$ Institute of Surgical Pathology, Department of Pathology, \\ Medical Center - University of Freiburg, Freiburg, Germany; \\ ${ }^{5}$ Institute for Medical Biometry and Statistics, Medical Center - University of Freiburg, Freiburg, Germany; \\ ${ }^{6}$ Department of Obstetrics and Gynecology, Medical Faculty Hospital Pilsen, \\ Charles University Prague, Plzen, Czech Republic; \\ ${ }^{7}$ Department of Pathology, Maastricht University Medical Center, Maastricht, the Netherlands; \\ ${ }^{8}$ Department of Gynecology and Obstetrics, University Medical Center RWTH Aachen, Aachen, Germany; \\ ${ }^{9}$ Institute of Veterinary Medicine, Georg-August-University Goettingen, Goettingen, Germany
}

\begin{abstract}
Background/Aim: The pro-angiogenic Cyr61 protein has been associated with tumorigenesis and cancer progression in different gynecological carcinomas. In this study, we evaluated the potential impact and clinical relevance of Cyr61 expression in patients with primary non-metastatic cervical cancer (CC). Patients and Methods: Cyr61 expression was assessed in tissue specimen of 48 patients with primary CC by immunohistochemical analysis. Expression levels were scored and correlated to clinico-pathological factors and outcome data. Results: High Cyr61 expression levels were present in $54.2 \%$ of CC tissues. Associations with histological grade $(p=0.030)$, depth of tumor invasion $(p=0.007)$ and GOG score $(p=0.027)$ were observed. Patients who overexpressed Cyr61 displayed an increased death rate (30.8\% vs. $18.2 \%)$ and a decreased 5-year-survival (76.9\% vs. 86.4\%). Conclusion: Our data indicate a potential functional impact of Cyr61 in development and the progression of CC. The definite tumor-relevant function (suppressive/promoting)
\end{abstract}

This article is freely accessible online.

Correspondence to: Sebastian Mayer, Department of Obstetrics and Gynecology, Medical Center - University of Freiburg, Hugstetterstr. 55, 79106 Freiburg, Germany. Tel: +49 76127030010, e-mail: sebastian.mayer@uniklinik-freiburg.de

Key Words: Cyr61, cervical cancer, hTra2 $\beta$, overall survival, histologic grade. of Cyr61 in CC and the prognostic relevance of Cyr61 overexpression has to be evaluated in larger cohorts.

The immediate-early gene Cysteine-rich 61 (Cyr61) was first described by O'Brien et al. in 1990 providing a first informative basis to adumbrate its potential role as functional jack-of-all-trades (1). An ever increasing number of studies in the past decades revealed the multiplicity of Cyr61's biological role, particularly with regard to its various physiological and pathological features in humans. During embryonic development Cyr61 regulates a multitude of crucial processes such as neovascularization (2) or chondrogenesis (3). Based on an exceeding variety of Cyr61 binding partners (receptors) such as integrins, insulin-like growth factors (IGFs), vascular endothelial growth factor (VEGF), LDL receptor proteins (LPRs), transforming growth factor $\beta$ (TGF- $\beta$ ), heparan sulphate proteoglycans (HSPGs) etc., the protein is involved in a vast number of signaling pathways regulating (neo)angiogenesis, inflammation, bone fracture and wound repair (4). Corresponding to its physiological properties, Cyr61 protein activity also exerts comparable functional characteristics in pathological settings, such as tumorigenesis and cancer metastasis in a range of different malignant organ neoplasms (4). Due to Cyr61 regulatory activity the protein promotes important tumor-associated cellular functions like adhesion, migration or epithelial to mesenchymal transition (EMT) (5). In previous studies, our group identified a micro-environmentdependent expression regulation mechanism of Cyr61 in 
human breast cancer $(6,7)$.

Cyr61 expression has been associated with tumorigenesis and cancer progression in certain gynecological carcinomas. In ovarian cancers aberrant Cyr61 expression on mRNA and protein level has been reported (8). The role of Cyr61 in endometrial cancer is not clear yet. Chien et al. found decreased levels of Cyr61 in endometrial cancer cell lines compared to normal endometrial cells and described increased apoptosis rates in endometrial cancer cells with forced Cyr61 overexpression (9). Watari et al. reported an overexpression of Cyr61 in $22.8 \%$ of endometrial carcinomas, which was associated with poorer OS (10).

The impact of Cyr61 in CC, which is still the fourth most common cancer in women accounting for an estimated 528,000 new cases and 266,000 deaths in 2012 worldwide (11), has not been investigated so far. To date CCN3 is the only member of the $\mathrm{CCN}$ protein family, which has been evaluated in CC. Zhang et al. reported an overexpression of $\mathrm{CCN} 3$ in $\mathrm{CC}$ tissue, which was associated with the stage of disease, the lymph node involvement and a decreased OS (12).

In the present study we intended to evaluate the potential impact and the clinical relevance of Cyr61 expression in patients with primary cervical carcinoma. Therefore Cyr61 expression levels were correlated to tumor characteristics and clinical data as well as to survival data.

\section{Patients and Methods}

Patients and treatment. Parrafin-embedded tumor specimens were obtained from 48 patients with invasive cervical carcinoma who treatment at the Department of Obstetrics and Gynecology, Medical Faculty Hospital, Pilsen, Czech Republic between 1988 and 1995. The study protocol was approved by the Institutional Review Board.

All patients were treated by radical hysterectomy and pelvic lymphadenectomy and staged according to FIGO (International Federation of Obstetricians and Gynecologists) classification of 1995. Pelvic radiotherapy was administered in consonance with the standards of the Medical Faculty Hospital, Pilsen, at that time.

Blinded to clinical data histological slides were reviewed by two experienced pathologists (Department of Pathology, Medical Faculty Hospital, Pilsen, Czech Republic) and tumor type, grading, stromal invasion, lymphatic vascular space invasion (LVSI) and pelvic lymph node status were determined. All tissue specimens and slides were re-evaluated by an experienced pathologist $(\mathrm{AzH})$ at the Department of Pathology, Medical Center - University of Freiburg, Germany, confirming the previous diagnoses

Patient's baseline and tumor characteristics including age, tumor type, tumor size, tumor grading, lymph node status, stromal invasion, LVSI and GOG score as well as follow-up data were obtained from the Department of Obstetrics and Gynecology, Medical Faculty Hospital, Pilsen, Czech Republic. Calculation of the GOG score was performed according to the propositions of the Gynecologic Oncology Group (13) by multiplying the relative risks for depth of tumor invasion, tumor size and LVSI. Patient's baseline and tumor characteristics are summarized in Table I.
Table I. Patient and tumor characteristics.

\begin{tabular}{|c|c|c|}
\hline Patient characteristics $(n=48)$ & $\mathrm{N}$ & $\%$ \\
\hline \multicolumn{3}{|l|}{ Age } \\
\hline$<50$ & 37 & 77.1 \\
\hline$>50$ & 11 & 22.9 \\
\hline \multicolumn{3}{|l|}{ FIGO stage } \\
\hline IB1 & 41 & 85.4 \\
\hline IB2 & 5 & 10.4 \\
\hline IIA & 2 & 4.2 \\
\hline \multicolumn{3}{|l|}{ Histologic tumor type } \\
\hline Squamous & 46 & 95.8 \\
\hline Adenocarcinoma & 1 & 2.1 \\
\hline Adenosquamous & 1 & 2.1 \\
\hline \multicolumn{3}{|l|}{ Tumor size (mm) } \\
\hline$\leq 35$ & 40 & 83.3 \\
\hline$>35$ & 8 & 16.7 \\
\hline \multicolumn{3}{|l|}{ Histological grade } \\
\hline G1 & 4 & 8.3 \\
\hline $\mathrm{G} 2$ & 28 & 59.3 \\
\hline G3 & 16 & 33.3 \\
\hline \multicolumn{3}{|l|}{ Depth of tumor invasion } \\
\hline$\leq 5 \mathrm{~mm}$ & 6 & 12.5 \\
\hline$>5 \mathrm{~mm}$ & 42 & 87.5 \\
\hline \multicolumn{3}{|l|}{ Pelvic lymph node status } \\
\hline No & 39 & 81.3 \\
\hline N1 & 9 & 18.8 \\
\hline \multicolumn{3}{|l|}{ LVSI } \\
\hline negative & 25 & 52.1 \\
\hline positive & 23 & 47.9 \\
\hline \multicolumn{3}{|l|}{ GOG score } \\
\hline$<40$ & 6 & 12.5 \\
\hline $40-120$ & 27 & 56.3 \\
\hline$>120$ & 15 & 31.3 \\
\hline
\end{tabular}

Immunohistochemistry. Five $\mu \mathrm{m}$ formalin-fixed and paraffin-embedded (FFPE) cervical carcinoma specimens were stained for Cyr61 and $\mathrm{hTra} 2 \beta$ protein. Antigen retrieval was performed for 10 minutes in a microwave oven at $600 \mathrm{~W}$ using AKO target Retrieval Solution High pH (K8004, DAKO Agilent Pathology Solutions, Agilent Technologies, Waldbronn, Germany). The endogenous peroxidase was blocked using the $\mathrm{H}_{2} \mathrm{O}_{2}$-Block FlexPeroxidase (EnVision ${ }^{\mathrm{TM}}$, DAKO, Dako Deutschland GmbH, Hamburg, Germany) for $10 \mathrm{~min}$ at room temperature. After incubation with primary antibody (Cyr61 H-78 sc13100 (Santa Cruz Biotechnology Inc., Heidelberg, Germany), 1:500; hTra2 $\beta$ provided by Prof. Stefan Stamm UCK, 1:5,000 (14)) at room temperature overnight. Incubation with ImmPRESS ${ }^{\mathrm{TM}}$ HRP universal IgG Peroxidase Polymer Detection Kit (MP-7500, VectorLabs, Peterborough, UK) was performed for $30 \mathrm{~min}$. For visualization ImmPACT DAB Peroxidase Substrate (SK-4105, VectorLabs) was used for $10 \mathrm{~min}$. PBS washing was followed by counterstaining with Mayer's Haemalaun solution (\#109249, Merck, Darmstadt, Germany). All slides were dehydrated in ascending alcohol concentrations and tissue slides were coversliped and mounted with Entellan new rapid mounting medium (\#107961, Merck).

Assessment of Cyr61 and hTra2 $\beta$ expression. Cyr61 and hTra $2 \beta$ expression was evaluated by an experienced pathologist (STB). Immunohistochemical staining was considered positive, if a brown 


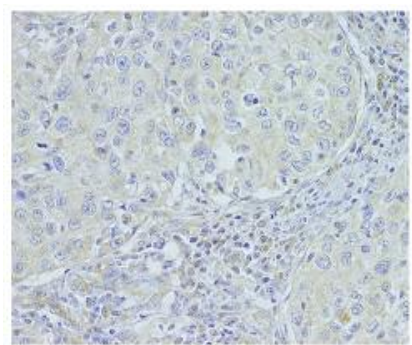

A

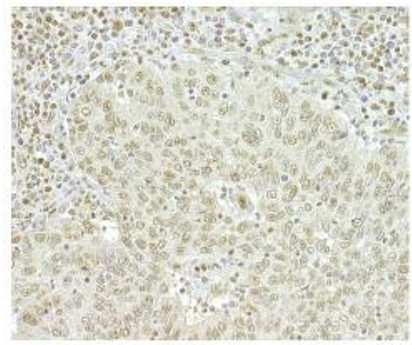

D

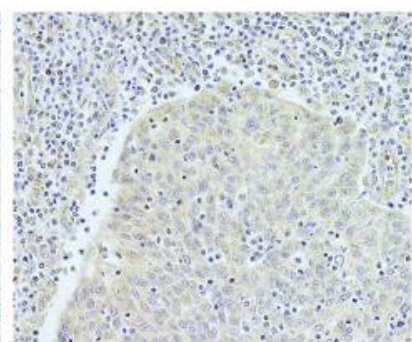

B

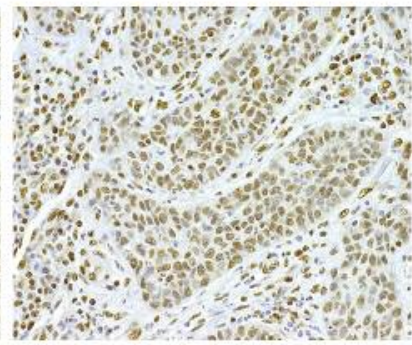

E

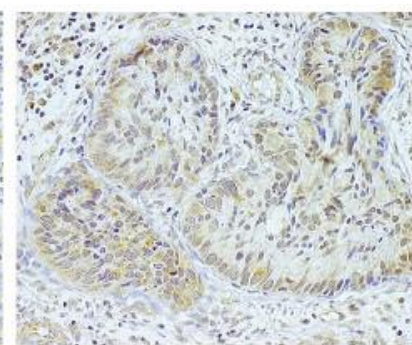

C

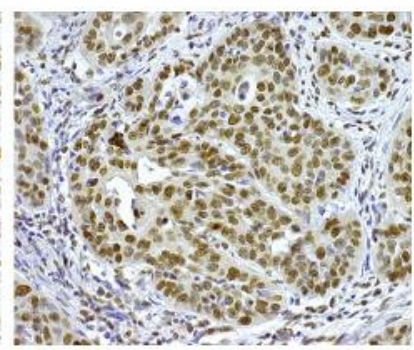

$\mathbf{F}$

Figure 1. Immunohistochemical analysis of Cyr61-and hTra2 $\beta$-protein expression in cervical cancer. Specific nuclear and cytoplasmatic expression of Cyr61 (A: weak expression, score $1+;$ B: moderate expression, score $2+$; $C$ : strong expression, score $3+$ ) and hTra2 $\beta$ (D: weak expression, score $1+$; E: moderate expression, score $2+;$ F: strong expression, score $3+$ ) in cervical carcinomas. Cyr61 antibody H-78 sc-13100 (Santa Cruz. Biotechnology), 1:500; hTra2 $\beta$ antibody provided by Prof. Stefan Stamm, UCK, 1:5000. Counterstaining with hemalaun. Magnification: $x 400$.

Table II. Univariable correlation analysis for Cyr61 and hTra2ß.

\begin{tabular}{lcrr}
\hline Variable1 & Variable2 & Correlation coefficient (Spearman's rho) & $p$-Value \\
\hline Cytoplasmatic Cyr61 overexpression & Histological grade & -0.324 & 0.024 \\
Nuclear Cyr61 expression & Histological grade & 0.360 & 0.012 \\
Cytoplasmatic hTra2 $\beta$ expression & Nuclear hTra2 $\beta$ expression & -0.365 & 0.014 \\
Cytoplasmatic hTra2 $\beta$ expression & Nuclear hTra2 $\beta$ overexpression & -0.297 & 0.047 \\
Cytoplasmatic hTra2 $\beta$ expression & Histological grade & 0.266 & 0.071 \\
\hline
\end{tabular}

signal was seen in the cytoplasmatic or in the nuclear compartment. A score was applied to classify the intensity of IHC staining as absent $(0)$, weak $(1+)$, moderate $(2+)$ or strong $(3+)$. For cytoplasmatic Cyr61 score 1 was categorized as low expression and score 2 and 3 were categorized as overexpression. For nuclear hTra2 $\beta$ score 1 and 2 were classified as low expression and score 3 was classified as overexpression (Figure 1).

Statistical analysis. Correlations of Cyr61 and hTra2 $\beta$ to clinicopathological factors were calculated using Spearman's rho. According to AIC criteria (15), logistic regression analyses were performed and a model with low standard deviations and with a low Akaike's Information criterion (AIC) including age, histologic grade 2 and 3 , tumor stage IB2 and IIA, depth of invasion, tumor size, GOG score, LVSI positive, cytoplasmatic hTra $2 \beta$ expression score 1 und nuclear hTra2 $\beta$ expression score 2 and 3 was selected. For analysis of overall survival the Kaplan-Meier estimator and the Cox model were used.

\section{Results}

Descriptive statistics. Forty-eight patients with histologically confirmed diagnosis of primary non-metastatic invasive cervical carcinoma who received surgical treatment at the Department of Obstetrics and Gynecology, Medical Faculty Hospital, Pilsen, Czech Republic between 1988 and 1995 were included in this study. Median age of patients was 41 years at the time of diagnosis (range $=24-68$ years). The characteristics of the study population are summarized in Table I.

Immunohistochemistry. Cyr61 expression was present in all evaluated tumor samples $(n=48)$. Cytoplasmatic Cyr61 expression (48 of $48=100 \%$ ) was more often observed than nuclear Cyr61 expression (14 of 48=29.2\%). Twenty-six 
Table III. Multivariable regression model for cytoplasmatic Cyr61 overexpression (AIC 64.87)

\begin{tabular}{lccc}
\hline Variable & $\begin{array}{c}\text { Regression } \\
\text { coefficient }\end{array}$ & $\begin{array}{c}95 \% \text {-confidence- } \\
\text { interval }\end{array}$ & $p$-Value \\
\hline Intercept) & -3.639 & $-10.671-3.393$ & 0.310 \\
Age & 0.042 & $-0.052-0.136$ & 0.385 \\
Histologic grade 2 & -2.435 & $-6.744-1.874$ & 0.268 \\
Histologic grade 3 & -5.473 & $-10.417--0.530$ & 0.030 \\
Tumor stage IB2 & -2.518 & $-5.338-0.302$ & 0.080 \\
Tumor stage IIA & -0.271 & $-4.863-4.322$ & 0.908 \\
Depth of invasion & 0.915 & $0.247-1.584$ & 0.007 \\
Tumor size & 0.039 & $-0.093-0.171$ & 0.561 \\
GOG score & -0.064 & $-0.119--0.010$ & 0.021 \\
LVSI positive & 2.602 & $-0.182-5.388$ & 0.067 \\
cytoplasmatic hTra2 $\beta$ score 1 & -0.060 & $-2.224-2.105$ & 0.957 \\
Nuclear hTra2 $\beta$ score 2 & -0.332 & $-3.149-2.486$ & 0.818 \\
Nuclear hTra2 $\beta$ score 3 & 0.648 & $-2.136-3.432$ & 0.648 \\
\hline
\end{tabular}

Table IV. Five-year overall survival (OS) stratified for Cyr61 expression.

\begin{tabular}{lccccc}
\hline Factor & Time & Score & N. at risk & Events & OS \\
\hline $\begin{array}{lcccc}\text { Cyr61 } \\
(\mathrm{n}=48)\end{array}$ & Baseline & All & 48 & 0 & $100.0 \%$ \\
& & Score 1 & 22 & 0 & $100.0 \%$ \\
& & Score 2 + 3 & 26 & 0 & $100.0 \%$ \\
& & & & \\
& \multirow{2}{*}{60 months } & All & 40 & 9 & $81.2 \%$ \\
& & Score 1 & 20 & 3 & $86.4 \%$ \\
& & Score 2 + 3 & 21 & 6 & $76.9 \%$ \\
\hline
\end{tabular}

tumor samples (54.2\%) revealed overexpression of cytoplasmatic Cyr61 compared to 22 tumor samples (45.8\%) with low cytoplasmatic Cyr61 expression. Only six tumor samples (12.5\%) showed high Cyr61 expression levels in the nuclear compartment compared to 8 tumor samples (16.7\%) with low and 34 tumor samples $(70.8 \%)$ without nuclear Cyr61 expression.

Qualitative and quantitative hTra2 $\beta$ expression was also determined in the cohort of $48 \mathrm{CC}$ specimen. Either no (37 of $48,77.1 \%$ ) or weak (score $1 ; 11$ of $48,22.9 \%$ ) cytoplasmatic hTra2 $\beta$ expression was observed. In contrary, overexpression of nuclear hTra2 $\beta$ was seen in $53.3 \%$ of tumor samples ( 24 of 45 ) compared to $46.7 \%$ with weak nuclear expression of hTra2 $\beta$ ( 21 of 45 ).

Univariable correlation analysis revealed a negative association between cytoplasmatic Cyr61 overexpression and histological grade (Spearman's correlation coefficient, rho $=-0.324 ; p=0.024)$ whereas nuclear Cyr61 expression

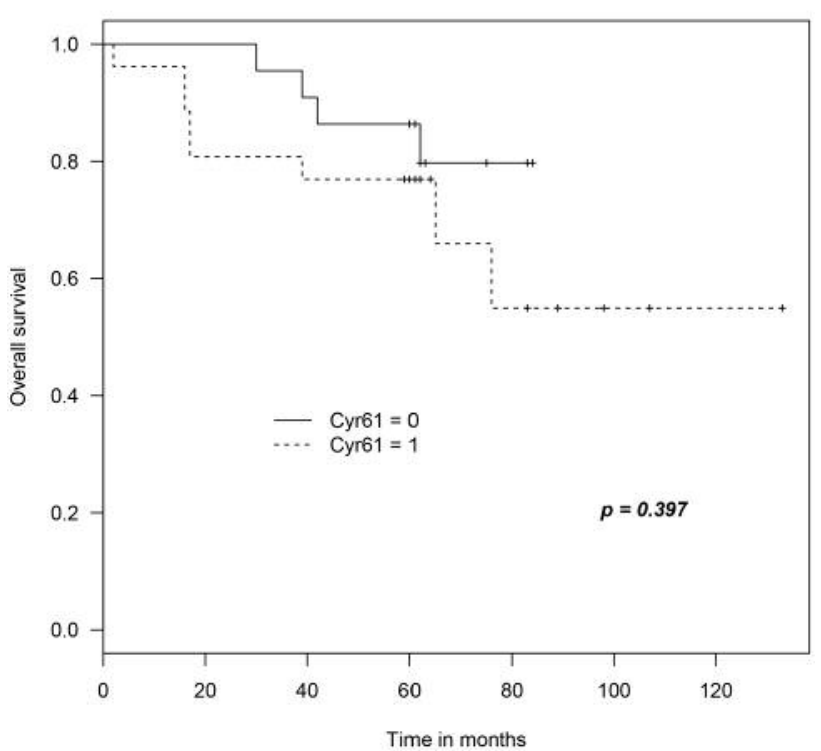

Figure 2. Overall survival (OS) with regard to Cyr61 expression. Survival analysis. Kaplan-Meier curves of patients with primary BC stratified for Cyr61 expression (Group 0: Score 1, Group: Score 2 and 3).

was positively associated with histological grade $(\mathrm{rho}=0.360$; $p=0.01$ ). Cytoplasmatic hTra2 $\beta$-expression was found to be inversely associated with nuclear $\mathrm{hTra} 2 \beta$ expression (rho $=-0.365, p=0.014)$ and nuclear hTRA $\beta$ overexpression $($ rho $=-0.297, p=0.047)$. Additionally, a trend towards a positive association of hTra2 $\beta$ with histological grade became apparent $(\mathrm{rho}=0.266 ; p=0.071)$. Further associations between Cyr61 or hTra2 $\beta$ and clinico-pathological parameters (e.g. histological tumor type, pelvic lymph node status, LVSI, depth of tumor invasion and GOG) were not observed in univariable analysis (Table II).

Multivariable logistic regression. Different models were screened for a suitable approach in the multivariable logistic regression analysis. The finally selected regression model with low estimates and with a low AIC (64.87) included the variables of age, histological grade, tumor size, tumor stage, depth of tumor invasion, LVSI, GOG score and cytoplasmatic, as well as nuclear hTra2 $\beta$ expression. Multivariable logistic regression analysis confirmed the negative association between cytoplasmatic Cyr61 overexpression and tumor grade $3(p=0.030)$ and showed a positive association with depth of tumor invasion $(p=0.007)$, as well as a negative association with the GOG score $(p=0.021)$. For LVSI $(p=0.067)$ and tumor stage $2(p=0.080)$ associations with cytoplasmatic Cyr61 overexpression were observed in this model, but statistical significance was not reached for both items (Table III). 
Survival analysis. Survival data of all 48 patients with known Cyr61 status were available. Median follow-up time was 62 months (range=2-133 months). During follow-up, 11 recurrences and 12 deaths were observed. Five-year-OS was $81.2 \%$.

Stratification of patients according to Cyr61 status showed a higher death rate ( 8 of $26=30.8 \%$ vs. 4 of $22=18.2 \%$ ) and a lower 5-year OS $(76.9 \%$ vs. $86.4 \%)$ in the patient subgroup with cytoplasmatic Cyr61 overexpression compared to the patients with low cytoplasmatic Cyr61 expression. Nevertheless, based on the Cox model, a significant association of cytoplasmatic Cyr61 overexpression and OS could not be detected ( $p=0.397)$ (Figure 2).

Subgroup analysis according to hTra $2 \beta$ status also revealed a higher death rate for patients with nuclear hTra $2 \beta$ overexpression ( 8 of $24=33.3 \%$ vs. 4 of $21=19.0 \%$ ) compared to patients with low nuclear hTra2 $\beta$ expression, but no difference in 5-year OS rates $(79.2 \%$ vs. $81.0 \%)$ could be determined between both subgroups (Table IV). This observation was confirmed by the Cox survival analysis which indicated no influence of nuclear hTra2 $\beta$ overexpression on OS ( $p=0.551)$ (data not shown).

\section{Discussion}

In the present study, based on 48 patients with primary $\mathrm{CC}$, Cyr61 expression was localized in the cytoplasm of all specimens $(100 \%)$ and in $29.2 \%$ in the nuclei of tumor samples. Overexpression of cytoplasmatic Cyr61 was present in the majority of tumors $(54.2 \%)$, whereas nuclear Cyr61 overexpression was only seen in $12.8 \%$ of tumors. We observed a negative association of cytoplasmatic Cyr61 overexpression and histological grade. This finding was consistent in univariable ( $p=0.024)$, as well as in multivariable $(p=0.030)$ analysis. Furthermore, multivariable regression analysis revealed associations of cytoplasmatic Cyr61 overexpression with depth of tumor invasion $(p=0.007)$ and GOG score $(p=0.027)$, as well as trends towards an association with tumor stage $2(p=0.080)$ and LVSI $(p=0.067)$. The observed associations for Cyr61 in CC display a heterogeneous picture so far. While the negative associations with histologic grade and GOG score may point to a more tumor suppressive function of Cyr61, the positive association of Cyr61 overexpression with tumor invasion and the trend for a positive association with LVSI account for a more tumor promoting role of Cyr61 in the development and progression of CC. This hypothesis is also supported by the outcome data of our patient cohort, which showed a higher death rate $(30.8 \%$ vs. $18.2 \%)$ and a decreased 5-year OS (76.9\% vs. $86.4 \%)$ in patients with Cyr61 overexpression. However, the observed trend in regard to survival did not reach statistical significance, which might be due to the relatively small patient cohort and the high number of censored patient cases at the end of the observation.
Our outcome data are in accordance with the findings of Cyr61 in other cancer entities. Watari et al. and Jeong et al. reported that Cyr61 overexpression was associated with poor prognosis in patients with endometrial and colorectal cancer $(10,16)$.

In the present study, cytoplasmatic Cyr61 expression did not correlate with nuclear hTra2 $\beta$ expression. This observation is in contrast to previous findings of our working group which could demonstrate that Cyr61 expression in gynecologic and breast cancer cell lines is regulated by hTra $2 \beta$ expression and acidosis (6). A possible explanation for these differing observations could be different microenvironmental conditions since the present results were derived from tissue specimen of patients whereas the previous study was performed with cell lines in vitro.

Several novel factors are under consideration to gain importance as prognosticators in CC diagnosis and therapy. For instance, in early-stage $\mathrm{CC}$ the protein TIMELESS could be associated with poor clinical outcome (17). Similarly, the nuclear receptor subfamily 2 group F member 6 (NR2F6) was identified as prognosticator for pelvic lymph node metastasis and poor outcome (18). The expression of Metadherin (MDTH) in CC tissues correlated with a higher risk of lymph node metastasis (19). Focusing on Cyr61-associated interactors, certain studies account for a signaling network in $\mathrm{CC}$ progression that clearly features the functional importance of Cyr61 activity. Song et al. point out interleukins IL-1 and IL6 as verisimilar valuable biomarkers and even potential therapeutic targets for CC (20). Most interestingly, Cyr61 is a known inducer of both factors, IL-1 (21) and IL-6 (22). In recent years the prognostic value of microRNAs in cervical carcinoma was subjected (23). In cervical carcinogenesis, Xie et al. were able to identify a regulatory influence of miR-205 (microRNA-205) triggering Cyr61 down-regulation in CC tissues (24).

This is the first study to evaluate the expression and clinical impact of Cyr61 in CC. The present analysis identified Cyr61 overexpression in the majority of CCs which is associated with clinico-pathological factors. The specific impact of Cyr61 in CC is not clear yet due to the heterogeneous associations of Cyr61 in the study population investigated. Nevertheless, the observed survival trend with decreased OS in patients with Cyr61 overexpression points to a more tumor-promoting role of Cyr61 in CC. Our results strengthen the importance of Cyr61 as an interesting target for further investigations to elucidate the suppressive or promoting impact and prognostic relevance of its expression in development and progression of $\mathrm{CC}$.

\section{Conflicts of Interest}

The Authors report no conflicts of interest. None of the authors received funding for this study. The Authors alone are responsible for the content and writing of the paper. 


\section{Acknowledgements}

The Authors thank Herta Bettendorf for technical support and Prof. Stefan Stamm for providing the hTra2 $\beta$ antibody.

\section{References}

1 O'Brien TP, Yang GP, Sanders L and Lau LF: Expression of cyr61, a growth factor-inducible immediate-early gene. Mol Cell Biol 10(7): 3569-3577, 1990.

2 Chen Y and Du XY: Functional properties and intracellular signaling of ccn1/cyr61. J Cell Biochem 100(6): 1337-1345, 2007.

3 O'Brien TP and Lau LF: Expression of the growth factorinducible immediate early gene cyr61 correlates with chondrogenesis during mouse embryonic development. Cell Growth Differ 3(9): 645-654, 1992.

4 Li J, Ye L, Owen S, Weeks HP, Zhang Z and Jiang WG: Emerging role of ccn family proteins in tumorigenesis and cancer metastasis (review). Int J Mol Med 36(6): 1451-1463, 2015.

5 Haque I, Mehta S, Majumder M, Dhar K, De A, McGregor D, Van Veldhuizen PJ, Banerjee SK and Banerjee S: Cyr61/ccn1 signaling is critical for epithelial-mesenchymal transition and stemness and promotes pancreatic carcinogenesis. Mol Cancer 10: 8,2011 .

6 Hirschfeld M, Jaeger M, Buratti E, Stuani C, Grueneisen J, Gitsch G and Stickeler E: Expression of tumor-promoting cyr61 is regulated by htra2-beta1 and acidosis. Hum Mol Genet 20(12): 2356-2365, 2011.

7 Hirschfeld $M$, zur Hausen A, Bettendorf $H$, Jager $M$ and Stickeler E: Alternative splicing of cyr61 is regulated by hypoxia and significantly changed in breast cancer. Cancer Res 69(5): 2082-2090, 2009.

8 Gery S, Xie D, Yin D, Gabra H, Miller C, Wang H, Scott D, Yi WS, Popoviciu ML, Said JW and Koeffler HP: Ovarian carcinomas: Ccn genes are aberrantly expressed and ccn1 promotes proliferation of these cells. Clin Cancer Res 11(20): 7243-7254, 2005.

9 Chien W, Kumagai T, Miller CW, Desmond JC, Frank JM, Said JW and Koeffler HP: Cyr61 suppresses growth of human endometrial cancer cells. J Biol Chem 279(51): 53087-53096, 2004.

10 Watari H, Xiong Y, Hassan MK and Sakuragi N: Cyr61, a member of ccn (connective tissue growth factor/cysteine-rich $61 /$ nephroblastoma overexpressed) family, predicts survival of patients with endometrial cancer of endometrioid subtype. Gynecol Oncol 112(1): 229-234, 2009.

11 Ferlay J, Soerjomataram I, Dikshit R, Eser S, Mathers C, Rebelo M, Parkin DM, Forman D and Bray F: Cancer incidence and mortality worldwide: Sources, methods and major patterns in globocan 2012. Int J Cancer 136(5): E359-386, 2015.
12 Zhang T, Zhao C, Luo L, Xiang J, Sun Q, Cheng J and Chen D: The clinical and prognostic significance of cen 3 expression in patients with cervical cancer. Adv Clin Exp Med 22(6): 839-845, 2013.

13 Delgado G, Bundy B, Zaino R, Sevin BU, Creasman WT and Major F: Prospective surgical-pathological study of disease-free interval in patients with stage ib squamous cell carcinoma of the cervix: A gynecologic oncology group study. Gynecol Oncol 38(3): 352-357, 1990.

14 Stoilov P, Daoud R, Nayler O and Stamm S: Human tra2-beta1 autoregulates its protein concentration by influencing alternative splicing of its pre-mrna. Hum Mol Genet 13(5): 509-524, 2004.

15 Schwarz G: Estimating the dimensions of a model. Ann Stat 6: 461-464, 1978.

16 Jeong D, Heo S, Sung Ahn T, Lee S, Park S, Kim H, Park D, Byung Bae S, Lee SS, Soo Lee M, Kim CJ and Jun Baek M: Cyr61 expression is associated with prognosis in patients with colorectal cancer. BMC Cancer 14: 164, 2014.

17 Zhang W, He W, Shi Y, Zhao J, Liu S, Zhang F, Yang J, Xie C and Zhang Y: Aberrant timeless expression is associated with poor clinical survival and lymph node metastasis in early-stage cervical carcinoma. Int J Oncol 50(1): 173-184, 2016.

18 Niu C, Sun X, Zhang W, Li H, Xu L, Li J, Xu B and Zhang Y: $\mathrm{Nr} 2 \mathrm{f} 6$ expression correlates with pelvic lymph node metastasis and poor prognosis in early-stage cervical cancer. Int J Mol Sci 17(10): pii: E1694, 2016.

19 Hou Y, Yu L, Mi Y, Zhang J, Wang K and Hu L: Association of mtdh immunohistochemical expression with metastasis and prognosis in female reproduction malignancies: A systematic review and meta-analysis. Sci Rep 6: 38365, 2016.

20 Song Z, Lin Y, Ye X, Feng C, Lu Y, Yang G and Dong C: Expression of il-1alpha and il-6 is associated with progression and prognosis of human cervical cancer. Med Sci Monit 22: 4475-4481, 2016.

21 Behm B, Babilas P, Landthaler M and Schreml S: Cytokines, chemokines and growth factors in wound healing. J Eur Acad Dermatol Venereol 26(7): 812-820, 2012.

22 Lin J, Zhou Z, Huo R, Xiao L, Ouyang G, Wang L, Sun Y, Shen B, Li D and Li N: Cyr61 induces il-6 production by fibroblastlike synoviocytes promoting th17 differentiation in rheumatoid arthritis. J Immunol 188(11): 5776-5784, 2012.

23 Dai S, Lu Y, Long Y, Lai Y, Du P, Ding N and Yao D: Prognostic value of micrornas in cervical carcinoma: A systematic review and meta-analysis. Oncotarget 7(23): 35369-35378, 2016.

24 Xie H, Zhao Y, Caramuta S, Larsson C and Lui WO: Mir-205 expression promotes cell proliferation and migration of human cervical cancer cells. PLoS One 7(10): e46990, 2012.

Received March 13, 2017

Revised April 7, 2017

Accepted April 10, 2017 